\title{
Favipiravir effect against rabies infection
}

\author{
Akira Nishizono ${ }^{1}$, Kentaro Yamada ${ }^{1}$, Yousuke Furuta ${ }^{2}$, Takashi Komeno ${ }^{2}$ \\ ${ }^{1}$ Department of Microbiology, Oita University, Japan, ${ }^{2}$ Research Laboratories, Toyama Chemical, Japan
}

\section{Background}

Rabies is a zoonotic lethal encephalitis caused by the rabies virus (RABV), which is usually transmitted by animal bites. The case fatality rate for rabies remains almost $100 \%$, and there is still no means of cure after the onset of symptoms although the development attempt for drugs has been done for many years. Recently, Favipiravir (6-fluoro-3-hydroxy-2pyrazinecarboxamide) has been shown to be active against a broad range of RNA viruses and been expected to be a therapeutic drug for rabies. In this study, we evaluated the antiviral efficacy of T705 against RABV infection in vitro and vivo.

\section{Methods}

Antiviral activity against RABV was examined in mouse neuroblastoma cell lines, NA and N2a cells. Cells were inoculated with RABV at an MOI of 0.01 and incubated for $96 \mathrm{~h}$ in the presence of T705. The viral titers in the media were determined using a focus assay. The antiviral efficacy of T705 in 6-week-old female ddY mice was also evaluated. Mice were intramuscularly inoculated with 105 FFU of the street (wild) strain 1088 and were administered T705 (30, 100, or $300 \mathrm{mg} / \mathrm{kg} /$ day, p.o.) or $0.5 \%$ methylcellulose (control) daily for 7 or 14 days. The administration was commenced $1 \mathrm{~h}$ or 4 days post inoculation, or on the day when symptom onset was apparently observed.

Results

A 3-4 $\log 10$ reduction in RABV growth in N2a cells treated by T705 $(1 \mathrm{mM})$, but the activity was lower in NA cells than in N2a cells. We detected the lack of HPRT expression which is requisite for conversion T705 to its active form in NA cells and the activity partially restored by the complementation of the expression of hprt gene in NA cells. T705 significantly improved morbidity and mortality in RABV infected mice when orally administered at a dose of 300 $\mathrm{mg} / \mathrm{kg} /$ day, beginning $\mathrm{h}$ after inoculation. However, the survival of RABV infected mice was not significantly improved when T705 treatment was initiated 4 days after inoculation or after the onset of symptoms.

Conclusions

Our data suggested that T705 was active against RABV and has the potential to provide an alternative to rabies immunoglobulin in rabies post exposure prophylaxis. 